Atomistic and mesoscale simulations of free solidification in comparison

This article has been downloaded from IOPscience. Please scroll down to see the full text article.

2009 Modelling Simul. Mater. Sci. Eng. 17055006

(http://iopscience.iop.org/0965-0393/17/5/055006)

View the table of contents for this issue, or go to the journal homepage for more

Download details:

IP Address: 193.196.64.2

The article was downloaded on 07/05/2010 at 07:46

Please note that terms and conditions apply. 


\title{
Atomistic and mesoscale simulations of free solidification in comparison
}

\author{
Ilya Maltsev ${ }^{1}$, Alexandr Mirzoev ${ }^{1}$, Denis Danilov² ${ }^{2}$ and Britta Nestler ${ }^{2}$ \\ ${ }^{1}$ Physical Department, South-Ural State University, Lenin av. 76, 454080 Chelyabinsk, Russia \\ 2 Institute of Applied Research, Karlsruhe University of Applied Sciences, Moltkestrasse 30, \\ 76133 Karlsruhe, Germany
}

Received 15 July 2008, in final form 30 April 2009

Published 16 June 2009

Online at stacks.iop.org/MSMSE/17/055006

\begin{abstract}
Solidification of an undercooled Lennard-Jones system is considered by atomistic and mesoscale simulations. The influence of the parameters of a Nosé-Hoover thermostat on the temperature profile in the molecular dynamics box during the free solidification of the sample is analyzed. Direct comparison of the temperature profiles and of the interface dynamics in molecular dynamics with phase-field simulations is given.
\end{abstract}

\section{Introduction}

Progress in theory and modeling of complex microstructures during solidification of metals opens new directions in the development of a quantitative description of solidification phenomena. One of the directions is multiscale modeling by coupling of processes at widely different lengths, times and energy scales. In general, it is impossible to construct a physical model that adequately describes the evolution in multiscale systems in the whole range of length and time scales from the atomistic to the mesoscale level. For such a case, one can construct a hierarchy of models and approaches which describe the system in limited ranges of scales providing a matching between them.

This multiscale approach has been applied to the problem of dendritic growth in [1-4]. The example of dendritic growth shows that the shape and the velocity of a dendrite are very sensitive to the solid-liquid interfacial energy and to the kinetic coefficient. The usually small anisotropy of these interfacial parameters also plays a crucial role in defining the operation state of the dendrite. However, the precise values of those key parameters have so far remained unmeasured experimentally for many materials, and existing anisotropy measurements remain limited to a few transparent organic systems. Therefore, the properties of the solid-liquid interface have been computed by molecular dynamics (MD) on atomistic scales and then the computed values form input parameters for a phase-field model (PFM) $[5,6]$ describing the development of the dendrite on the mesoscale. The growth of the dendrite is accompanied by a permanent morphological instability of the solid-liquid interface (which actually leads to a complex branched structure) and therefore small variations of the input parameters can 
result in significant changes in the final microstructure during the simulations. Therefore, one needs to pay attention to the matching of data transferred between the levels of the multiscale framework.

The accuracy of MD simulations depends on a number of factors. Among the interatomic potential and the size (number of atoms) of the system, a number of simulation details play an important role, especially the time step of integration and the method of temperature control. In the pioneering work of Broughton et al [7] it has been mentioned that the release of latent heat leads to a nonuniform temperature profile around the crystal-melt interface. In [1-4] the Nosé-Hoover thermostat for temperature control has been used to keep the prescribed temperature in the box as whole and no discussion on the temperature profile in the system is given. A temperature profile was considered in detail in [7] but for a thermostat applied only to a part of the box.

We suppose that underestimation of the temperature control can lead to a number of errors in the modeling of solidification. The solidification dynamics depends on the local temperature $T$ at the crystal-melt interface which can exhibit significant deviations from the temperature $T^{\prime}$ of the applied thermostat. In section 2 we analyze the influence of the thermostat on the parameters of the solidification process in connection to the system size using the LennardJones interatomic potential. In section 3 we compare the MD results with PFM simulations in order to establish a matching between the models in the overlapping length and time scales.

\section{MD simulations of solidification}

\subsection{Method}

The atoms interact by the Lennard-Jones potential

$$
U=4 \epsilon\left(\left(\frac{\sigma}{r}\right)^{12}-\left(\frac{\sigma}{r}\right)^{6}\right)
$$

in the standard notation. We use dimensionless variables with length scale $\sigma$ and energy scale $\epsilon$, the mass of atoms $m=1$ and the Boltzmann constant $k_{\mathrm{B}}=1$. Correspondingly, the time is measured in $\sqrt{m \sigma^{2} / \epsilon}$ units and the temperature in units of $\epsilon$.

We apply to the system the Nosé-Hoover thermostat described in $[8,9]$. The equations of motion have the form

$$
\begin{aligned}
& \dot{\vec{r}}_{i}=\frac{\vec{p}_{i}}{m_{i}}, \\
& \dot{\vec{p}}_{i}=\vec{f}_{i}-\xi \cdot \vec{p}_{i}, \\
& \dot{\xi}=\frac{\left(\sum_{i} \frac{\vec{p}_{i}^{2}}{m_{i}}-(3 N+1) k_{\mathrm{B}} T_{0}\right)}{Q_{\text {temp }}}, \\
& Q_{\text {temp }}=3 N k_{\mathrm{B}} T_{0} \cdot \tau_{\text {temp }}^{2} .
\end{aligned}
$$

Here $\vec{r}_{i}$ and $\vec{p}_{i}$ are the coordinate and the momentum of $i$ th atom, $\vec{f}_{i}$ is the force acting on the atom, $N$ is the number of atoms in the system. The thermostat has the temperature $T_{0}$ and includes inertial parameter $Q_{\text {temp }}$ related to the relaxation time $\tau_{\text {temp }}$. The pressure is controlled by a Nosé-Hoover barostat [9].

The crystal and melt coexist in equilibrium at the temperature $T_{\mathrm{M}}=0.62$. To derive the melting temperatures we perform standard NPT ensemble (constant number of particles, 
constant pressure and constant temperature) simulations with the Nosé-Hoover thermostat to determine the equilibrium lattice parameter of the bulk crystal as a function of temperature at zero pressure. Using this estimation of $T_{\mathrm{M}}$, we equilibrate a crystal at the temperature and lattice constant corresponding to the initial estimation. In the next step, half of the atoms in the box are melted maintaining constant volume, and rest of the atoms are fixed. The melt is held at a temperature approximately 0.1 above $T_{\mathrm{M}}$ estimation. This procedure results in a periodic two phase solid-liquid setup containing two crystal-melt interfaces. Keeping the solid atoms fixed, the liquid atoms are equilibrated at the estimated melting temperature, allowing the length of the simulation box normal to the interfaces to adjust in order to minimize stress. This equilibration phase takes about 100 time units. After the equilibration, the entire system is allowed to relax employing canonical ensemble NVT up to 1000 time units at the temperature equal to the estimated value of $T_{\mathrm{M}}$. A refined estimation of $T_{\mathrm{M}}$ is then derived employing $\mathrm{NPH}$ ensemble with no thermostat. In these simulations the periodic dimensions parallel to the solid-liquid interfaces are held fixed, while the length of the box normal to the interfaces is allowed to change dynamically with zero imposed stress.

In the following section the model systems consist of 12000 and 106000 atoms in a 3D box with periodic boundary conditions in all the directions. The simulation box is elongated in the $z$-direction and has dimensions $5 \times 5 \times 130$ unit cells for 12000 atoms and $5 \times 5 \times 1140$ unit cells for 106000 atoms. The melt and crystals have been placed so that the directions [1 00 , $\left[\begin{array}{lll}0 & 1 & 0\end{array}\right]$, and $\left[\begin{array}{lll}0 & 0 & 1\end{array}\right]$ coincide with the axes of the coordinate system. Two opposite crystals with the melt inbetween form two crystal-melt interfaces with $\left(\begin{array}{lll}1 & 0 & 0\end{array}\right)$ orientation.

We present simulations with three values of the relaxation parameter $\tau_{\text {temp }}=0.7, \tau_{\text {temp }}=7$ and $\tau_{\text {temp }}=70$ at zero pressure and temperature $T_{0}=0.56$. The results show the comparison of temperature profiles in the system for different $\tau_{\text {temp }}$. The profiles are averaged over different configurations to reduce the fluctuations.

\subsection{Effect of thermostat}

Figure 1 shows the temperature profiles in the system of 12000 atoms for the three values 0.7 , 7 and 70 of the relaxation parameter $\tau_{\text {temp. }}$. All three temperature profiles correspond to an equal position of crystal-melt interfaces indicated by vertical lines at the left and right sides, i.e. the profiles correspond to different time intervals due to variation of the growth velocity as discussed further. The thermostat has the temperature 0.56 and the averaged temperature $\bar{T}$ in the system takes the values $\bar{T}=0.5592$ for $\tau_{\text {temp }}=0.7, \bar{T}=0.5611$ for $\tau_{\text {temp }}=7$ and $\bar{T}=0.6014$ for $\tau_{\text {temp }}=70$. In the case of $\tau_{\text {temp }}=70$, we can conclude that the relaxation is too slow to remove the released latent heat and the system is not in equilibrium with the thermostat. The temperature profiles for the first two values of $\tau_{\text {temp }}$ (the solid and dotted lines in figure 1) are similar and demonstrate an increase in local temperature at the interface above the thermostat temperature due to release of the latent heat, but the temperature of the melt is appreciable below the thermostat value of 0.56 . In contrast, the profile for $\tau_{\text {temp }}=70$ (dashed line) has the temperature above the thermostat in the melt, at the interfaces and in crystals. As a consequence, the temperature of the crystal-melt interfaces increases with increasing $\tau_{\text {temp }}$ and the measured growth velocity becomes a function of the relaxation parameter $\tau_{\text {temp }}$ as shown in figure 2.

The cause of hollow in the melt temperature is the thermostat, which maintains only the average temperature in the system, so the hot interfaces should be counterbalanced by the melt. The thermal diffusion is not enough to equalize the nonuniformity of the temperature. In the next step, we tested the larger simulation box with 106000 atoms. Figure 3 shows the evolution of the temperature profile when the thermostat with $\tau_{\text {temp }}=0.7$ is applied. In 


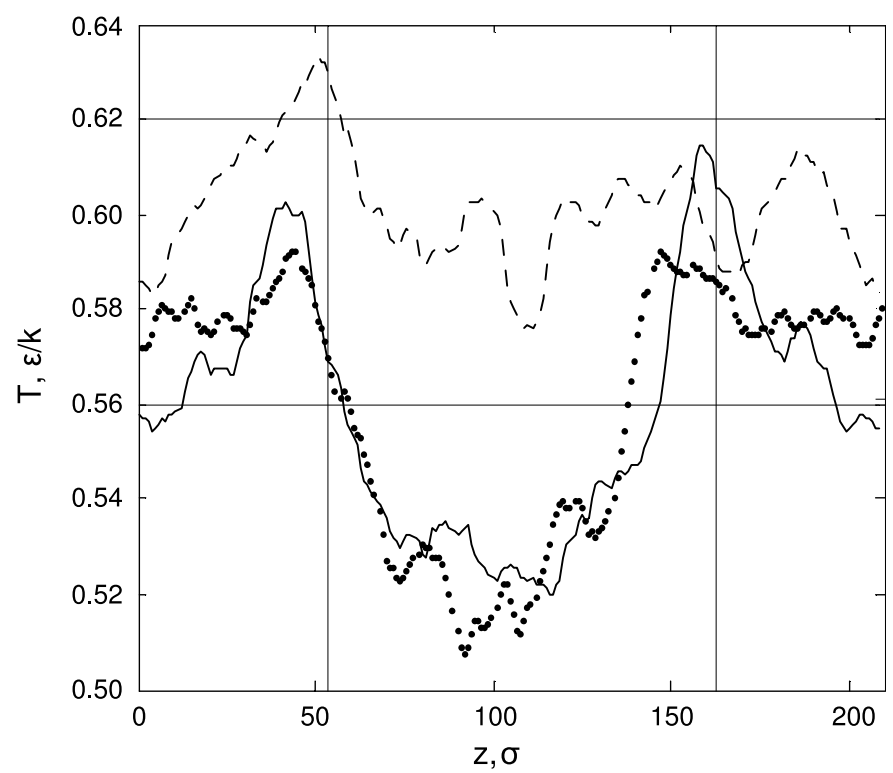

Figure 1. Temperature profiles in the system with 12000 atoms. The solid line corresponds to the relaxation time $\tau_{\text {temp }}=0.7$, the dotted line corresponds to $\tau_{\text {temp }}=7$ and the dashed line corresponds to $\tau_{\text {temp }}=70$. Vertical lines mark the positions of the crystal-melt interfaces. The top horizontal line shows the melting temperature and the bottom line shows the temperature of the thermostat.

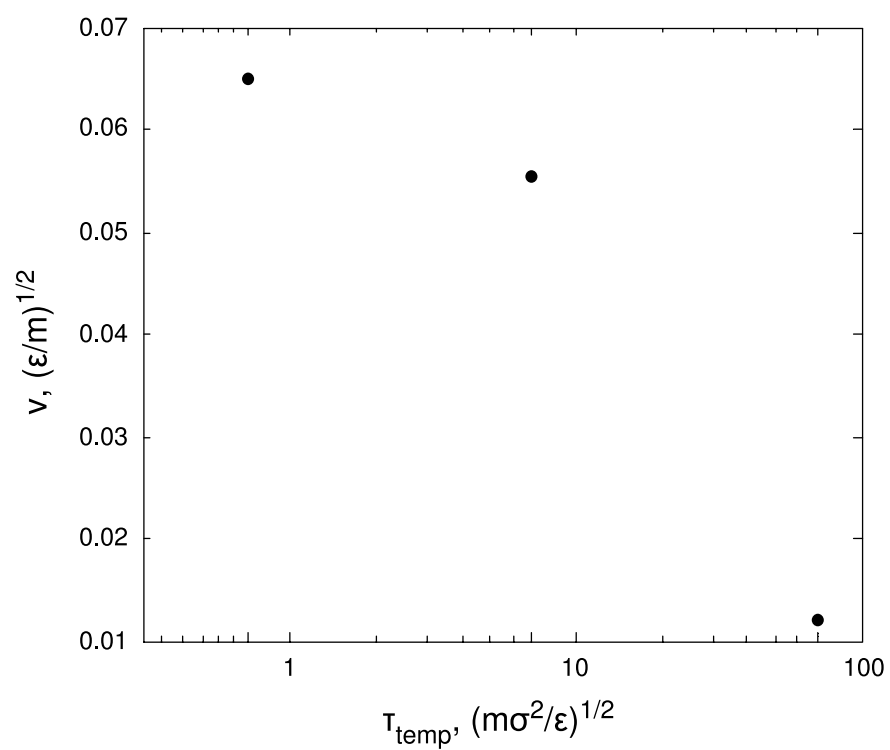

Figure 2. The growth velocity $v$ of the crystal-melt depending on parameter $\tau_{\text {temp }}$.

the initial stage of solidification, the temperature of the melt is equal to the temperature $T_{0}$ of the thermostat. The interfaces propagate into the melt and heated up atoms form the crystal. With the growth of the crystals, the fraction of the 'hot' regions increases and the temperature of the melt decreases to a values below $T_{0}$. 


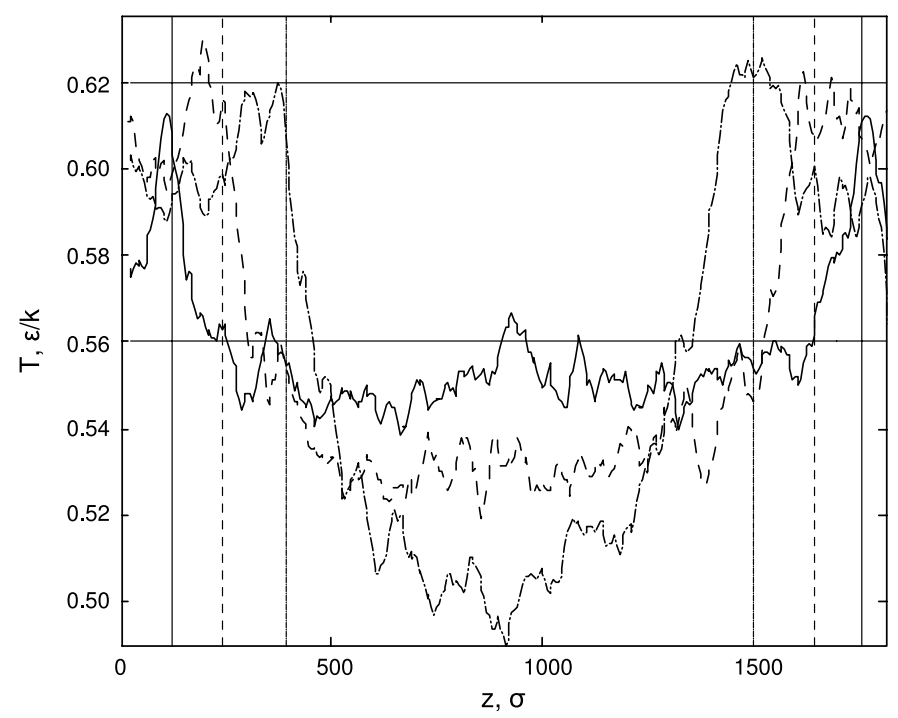

Figure 3. Temperature profiles in the system of 106000 atoms at different times: solid line$t=1500$, dashed line- $t=15000$ and dashed-dotted line $-t=30000$. The corresponding vertical lines show the positions of the crystal-melt interfaces. The horizontal lines mark the melting temperature (top) and the temperature of the thermostat (bottom).

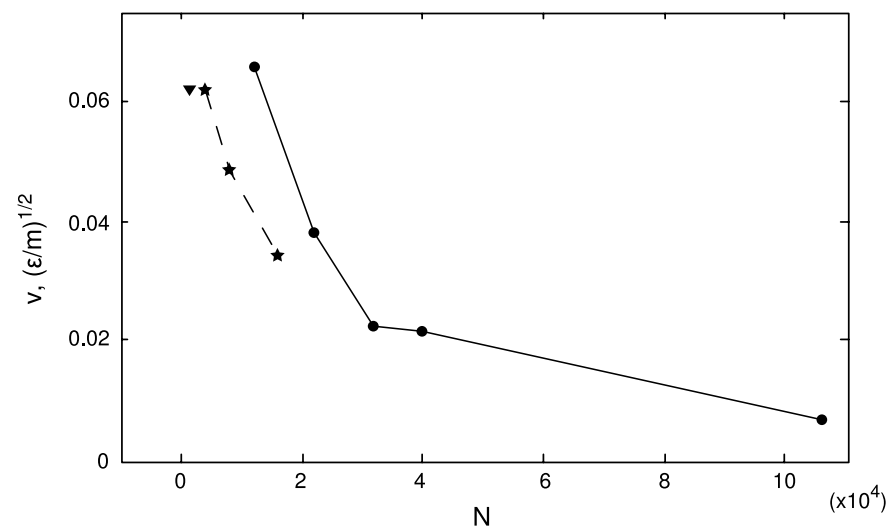

Figure 4. Dependence of the measured growth velocity $v$ on the size of the simulation box, i.e. on the number of atoms in the system for $\tau_{\text {temp }}=0.7$. The points show the results of this work. The stars show the data from [11], the triangle shows the data from [10].

As a consequence, the observed growth velocity depends on the size of the simulation box, figure 4. The growth velocity depends on the temperature of the interface, but the thermostat controls only the averaged temperature in the system. We have noted above that the released latent heat remains mainly in the crystal and increases the interface temperature. After some time, a regime of stationary growth with constant velocity can be reached as a balance between the release and the diffusion of the latent heat is established. Due to increase in the box size the balance occurs at higher temperatures of the interface and results in lower growth velocities (see figure 4). Similar results have been reported in $[10,11]$ and they are shown in figure 4 by the dashed line. 


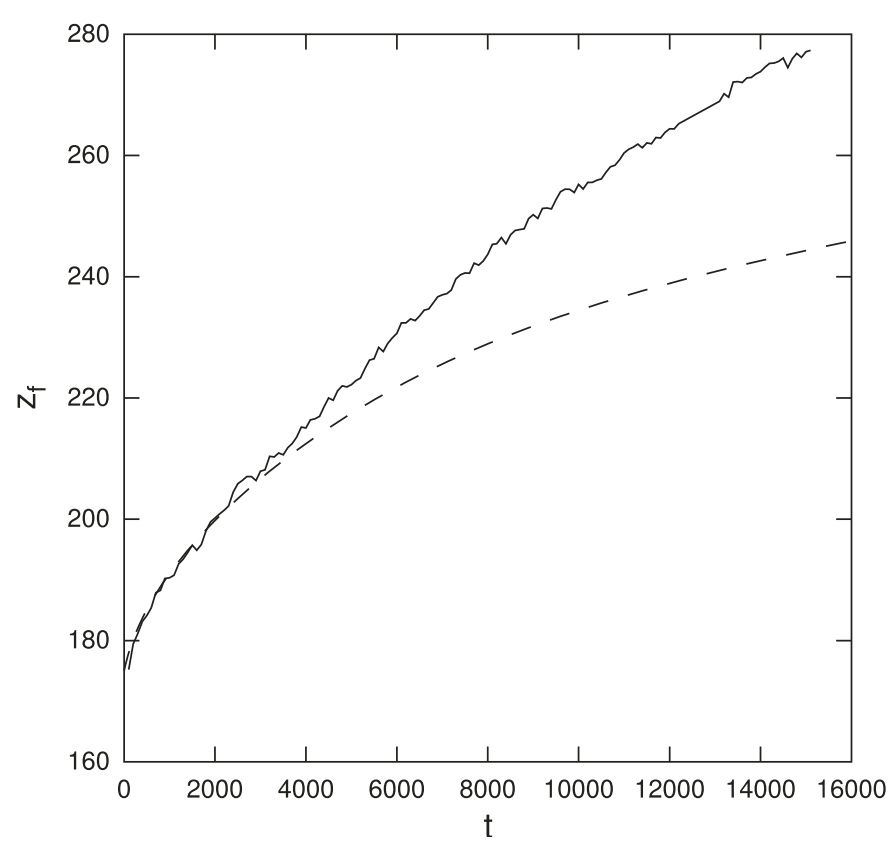

Figure 5. Position of the crystal-melt interface in time. The solid line shows the MD data, the dashed line shows the PFM simulations.

\section{Comparison of atomistic and mesoscale simulations}

The MD approach allows the detailed examination of the crystal-melt interface. However, the atomistic methods are limited by $10-100 \mathrm{~nm}$ on the length scale when the development of the morphological instability and the formation of dendrites occur on the length scales of order $1 \mu \mathrm{m}$. Therefore, when modeling on these scales, one needs to use mesoscopic models, for example a PFM [12]. Calculated by MD properties of the interface form a subset of input parameters in the mesoscopic model. Such an approach has been applied in $[5,6]$ where the results of MD simulations in [1] have been used as input parameters for the phase-field equations.

In this section we examine the congruence of atomistic and mesoscale simulations in the range of overlapping length scales. In order to minimize the effects described in section 2.2, the thermostat was applied only to narrow areas of $10 \times 10 \times 10$ in units of $\sigma$ on the left and right sides of the simulation box with size $10 \times 10 \times 680$ in units of $\sigma$, where the initial crystals are placed. In the rest of the box, the released latent heat can propagate by diffusion. To control the temperature in the selected areas, we use a rescaling of the velocities of the atoms at each time step. To the rest of the MD box no thermostat was applied. This configuration results in nonequilibrium nonisothermal crystallization after the system was undercooled to the initial temperature $T_{0}=0.57$ (also the temperature of the thermostat).

The method of the MD is described in section 2.1. The MD simulations will be compared with the results of the mesoscopic PFM which assumes a diffuse crystal-melt interface, that is in accordance with the structure of the interface in the MD simulations. The order parameter $0 \leqslant \varphi(z, t) \leqslant 1$ called the phase field describes the thermodynamic state of the system at location $z$ at the moment of time $t$ so that the value $\varphi=0$ corresponds to the melt, $\varphi=1$ corresponds to crystal phase and transition layer $0<\varphi<1$ approximates the diffuse interface. 


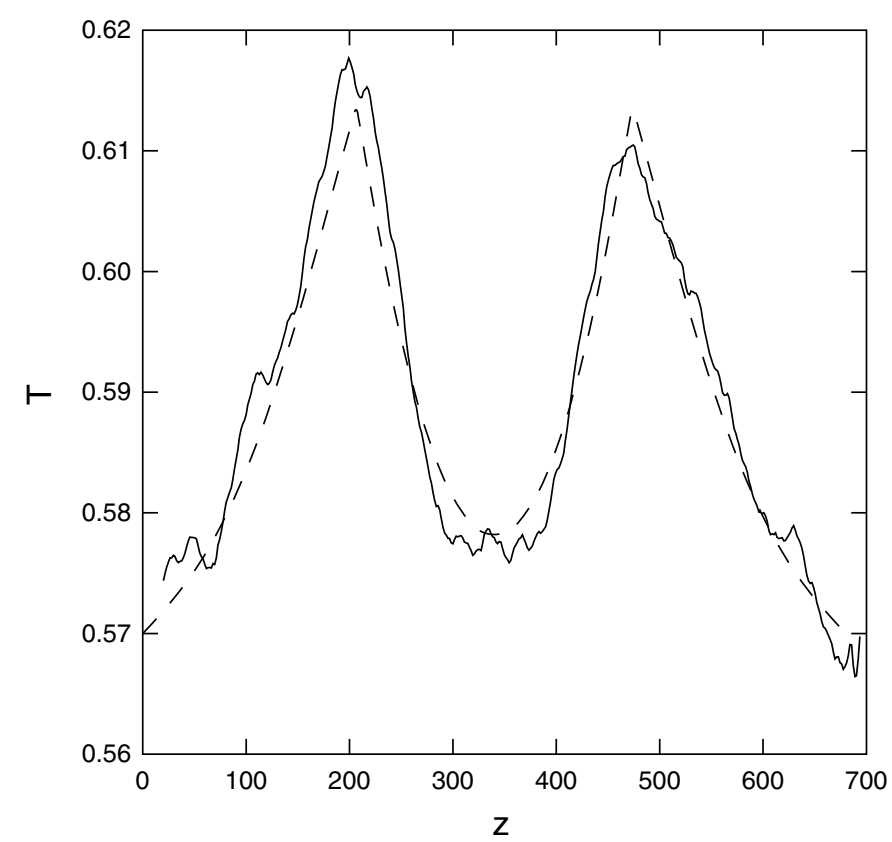

Figure 6. Temperature profiles at $t=3000$. The solid line shows the MD data, the dashed line shows the PFM simulations.

Taking into account the elongated geometry of the MD box, we write the evolution equations of the PFM in the form

$$
\begin{aligned}
& \frac{\partial T}{\partial t}=a \frac{\partial^{2} T}{\partial z^{2}}+\frac{Q}{c_{p}} \frac{\partial h(\varphi)}{\partial t}, \\
& \frac{1}{v} \frac{\partial \varphi}{\partial t}=\frac{\partial^{2} \varphi}{\partial z^{2}}-\frac{1}{2 \delta^{2}} \frac{\partial g}{\partial \varphi}-\frac{1}{2 \delta} \frac{Q}{\gamma} \frac{T-T_{\mathrm{M}}}{T_{\mathrm{M}}} \frac{\partial h}{\partial \varphi} .
\end{aligned}
$$

The dimensionless units of the Lennard-Jones potential have been used. The form of the equations, i.e. the last terms on the rhs of equations (6) and (7), corresponds to the approximation of the free-energy density used in [6] on multiscale modeling of dendritic growth in pure Ni. Therefore we start the comparison with MD from this form of the equations. The temperature field is described by the common heat equation with the thermal diffusivity $a$. The last term in equation (6) describes the release of the latent heat $Q, c_{p}$ is the heat capacity. The dynamics of the phase field is described by the partial differential eqaution (7) where $v$ is the interface mobility, $\delta$ is the parameter determining the thickness of the interface, $\gamma$ is the interfacial energy, and $T_{\mathrm{M}}$ is the equilibrium melting point. The local minima of the energy in the system are determined by the double-well potential $g(\varphi)=\varphi^{2}(1-\varphi)^{2}$ and the interpolation between the phases through the interface is given by the function $h(\varphi)=\varphi^{2}(3-2 \varphi)$.

In accordance with the MD setup, equations (6) and (7) are solved numerically in the domain $0 \leqslant z \leqslant 680 \sigma$ with the boundary conditions $T(0, t)=T(680, t)=0.57$. The initial temperature takes uniform value $T(z)=0.57$, and the initial phase-field profile has two solid layers on the left and right sides with the melt inbetween. 


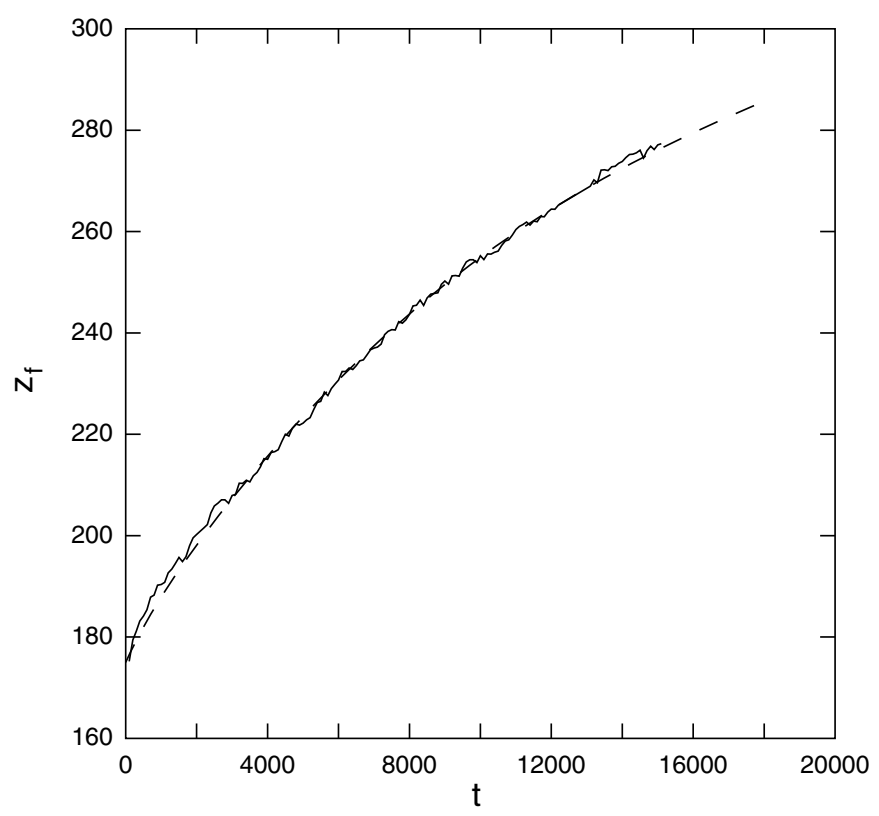

Figure 7. Position of the crystal-melt interface in time for modified equations with $Q(T)$. The solid line shows the MD data, the dashed line shows the PFM simulations.

The thickness of the diffuse interface can be determined from the MD data using an order parameter based on a structure factor [13]

$$
S(\vec{k})=\frac{1}{N} \sum_{i}\left|\mathrm{e}^{i^{k} \vec{r}}\right|,
$$

where $\vec{k}$ is the reciprocal lattice vector directed along the $(100)$ direction. The sum over all atoms falling within the local volume gives the value of the order parameter in this local volume. The structure factor takes the values approximately between 0.6 and 0.8 in the bulk crystal and values about 0 in the bulk melt. For an equilibrium interface, equation (7) has the solution

$$
\varphi_{0}(z)=\frac{1}{2}\left(1-\tanh \left(\frac{3 z}{2 \delta}\right)\right) .
$$

Using this solution we can determine the parameter $\delta$ by fitting the $\varphi_{0}(z)$ to the structure factor from MD simulations scaled to the range of values from 0 to 1 . The obtained value $\delta=2$ shows satisfactory agreement with MD results also for the nonequilibrium moving interface.

We use two characteristics of the crystallization process to examine the matching between the atomistic MD and the mesoscopic phase-field simulations. The first characteristics is the dynamics of the crystal-melt interface. The position of the interface is determined by the point where the structure factor or the phase field takes the intermediate value 0.5 . The second characteristics is the temperature profile. In the phase-field simulations it is determined by equation (6). In MD simulations the box is divided into local volumes with the local value of the temperature. To smooth the fluctuations, an average over different configurations is used.

In the phase-field simulations the following values of the parameters have been used: $T_{\mathrm{M}}=0.62, v=0.2, a=1.5, c_{p}=5.5, Q=1.1$ and $\gamma=0.374$. This set of parameters provides good agreement between interface dynamics and temperature profiles in PFM and 

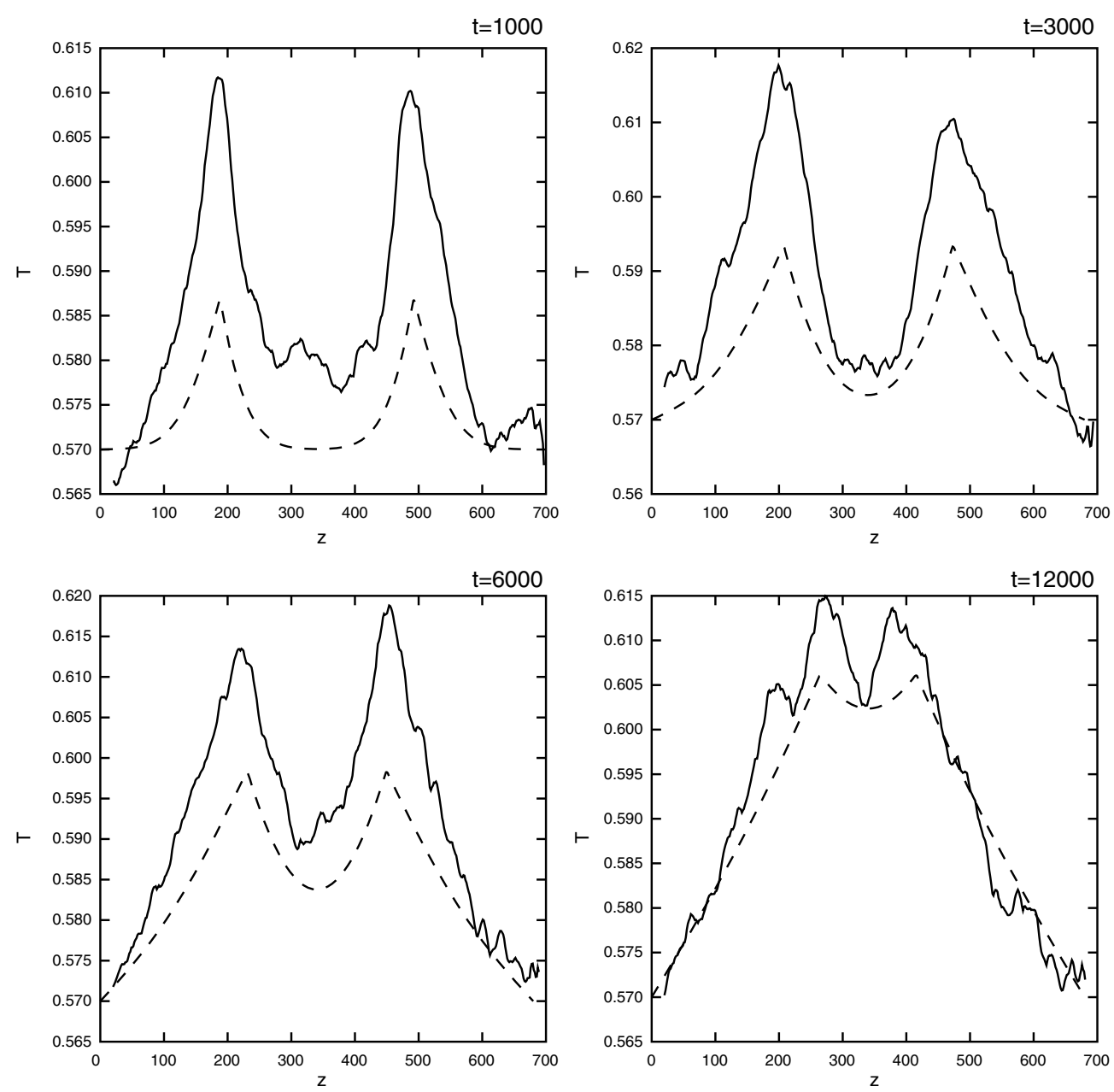

Figure 8. Temperature profiles in the system at different times. Solid lines show the MD data, dashed lines show the PFM simulations.

MD simulations in the time interval $0<t<4000$, figures 5 and 6. For $t>4000$ the temperature fields generated by the two interfaces begin to overlap and from this time the phase-field simulations predict a smaller interface velocity as obtained in MD simulations. On testing different values of the parameters in equations (6) and (7) we have found that the discrepancy at long times between the PFM and MD has a systematic characteristic and it cannot be eliminated by tuning the parameter set.

Therefore, we consider an extension of equations (6) and (7) by introducing a temperature dependence of latent heat $Q(T)=Q_{0}\left(1+k\left(T-T_{\mathrm{M}}\right)\right)$. Using the values $Q_{0}=0.472$, $\gamma=0.505, k=-3.46$ we can achieve good agreement for interface dynamics data in the whole range of the time, figure 7. However, a disagreement remains in the temperature profiles: the PFM predicts a lower temperature at the crystal-melt interface in comparison with the MD data, figure 8.

Thus we get a result that the PFM in the form of equations (6) and (7) does not provide a fully consistent match with the MD results. On the one hand, we can achieve an agreement 
on the description of the release of latent heat and of the temperature profile, but we get the deviation in the dynamics of the interface motion. On the other hand, by introducing the temperature dependent latent heat parameter into the equations, we can reach an agreement on the dynamics of the interface motion between the PFM and MD simulations, but it is accompanied by the deviation of temperature profiles. A possible reason for this behavior consists of specific mechanisms of release and of diffusion of the latent heat during the crystallization process which are included in the modeling on the atomistic level and are simplified out in the mesoscopic description. The identification and investigation of this point will be continued in subsequent work.

\section{Conclusions}

In this paper the crystallization of a pure Lennard-Jones system is modeled by atomistic MD and the mesoscopic PFM in comparison. The influence of the parameters of the NoséHoover thermostat on the temperature profile and on the growth velocity in MD simulations is investigated. The results show that qualitatively the crystallization occurs in the nonisothermal regime independent of the relaxation parameter of the thermostat. Correspondingly, the growth velocity observed in the MD simulations depends on the size of the MD box and on the relaxation parameter $\tau_{\text {temp }}$.

The MD results are compared with the phase-field simulations and the systematic deviation between the predictions of MD and PFM is observed. A correction of the phase-field formulation is necessary in order to match the atomistic and mesoscopic simulations in the range of overlapping scales within a framework of multiscale modeling.

\section{Acknowledgments}

This work was supported by the German Research Foundation (DFG) and by the Russian Foundation for Basic Research (RFBR). The authors gratefully acknowledge the funding. The authors also thank the two anonymous referees for their very useful comments.

\section{References}

[1] Sun D Y, Asta M and Hoyt J J 2004 Phys. Rev. B 69024108

[2] Sun D Y, Asta M and Hoyt J J 2004 Phys. Rev. B 69174103

[3] Hoyt J J and Asta M 2002 Phys. Rev. B 65214106

[4] Xia Z G, Sun D Y, Asta M and Hoyt J J 2007 Phys. Rev. B: Condens. Matter Mater. Phys. 75012103

[5] Hoyt J J, Asta M and Karma A 2003 Mater. Sci. Eng. R: Rep. 41 121-63

[6] Bragard J, Karma A, Lee Y H and Plapp M 2002 Interface Sci. 10 121-136

[7] Broughton J Q, Gilmer G H and Jackson K A 1982 Phys. Rev. Lett. 49 1496-500

[8] Nosé S 1984 J. Chem. Phys. 81 511-19

[9] Nosé S 1990 J. Phys.: Condens. Matter 2 SA115-9

[10] Briels W J and Tepper H L 1997 Phys. Rev. Lett. 79 5074-7

[11] Tepper H L and Briels W J 2001 J. Chem. Phys. $1159434-43$

[12] Boettinger W J, Warren J A, Beckermann C and Karma A 2002 Annu. Rev. Mater. Res. 32 163-94

[13] Chen E T, Barnett R N and Landman U 1989 Phys. Rev. B 40 924-32 